\title{
Two infinite families of critical clique-Helly graphs
}

\author{
Liliana Alcón a,b, Miguel Pizaña ${ }^{c}$, Gabriela Ravenna ${ }^{\mathrm{a}, \mathrm{b}, *}$ \\ a Dto. de Matemática, FCE-UNLP, La Plata, Argentina \\ ${ }^{\mathrm{b}}$ CONICET, Argentina

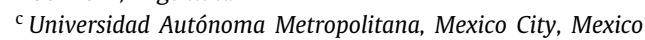

\section{A R T I C L E I N F O}

\section{Article history:}

Received 18 April 2018

Received in revised form 26 June 2019

Accepted 29 June 2019

Available online 22 July 2019

\section{Keywords:}

Helly property

Clique-Helly graphs

Clique graphs

\begin{abstract}
A B S T R A C T
A graph is clique-Helly if every family of pairwise intersecting (maximal) cliques has non-empty total intersection. Dourado, Protti and Szwarcfiter conjectured that every clique-Helly graph contains a vertex whose removal maintains it as a clique-Helly graph. We present here two infinite families of counterexamples to this conjecture.
\end{abstract}

(C) 2019 Elsevier B.V. All rights reserved.

\section{Introduction}

A set family $\mathcal{F}$ satisfies the Helly property if the intersection of all the members of every pairwise intersecting subfamily of $\mathcal{F}$ is non-empty. This property, originated in the famous work of Eduard Helly on convex sets in the Euclidean space, has been widely studied in diverse areas of theoretical and applied mathematics such as extremal hypergraph theory, logic, optimization, theoretical computer science, computational biology, databases, image processing and graph theory. A few surveys have been written on the Helly property, see for instance [2,4-6,8].

From the computational and algorithmic point of view, the relevance of the Helly property has been highlighted in the survey [5]. In the section Proposed Problems of that work, the authors posed the following open question:

Conjecture 1.1 (Dourado, Protti and Szwarcfiter [5]). Every clique-Helly graph contains a vertex whose removal maintains it as a clique-Helly graph.

In this work, we prove the conjecture is false: we will exhibit two infinite families of clique-Helly graphs $G$ such that $G-x$ (the graph obtained from $G$ by removing vertex $x$ ) is not clique-Helly for every vertex $x$ of $G$. Moreover, the family in Section 3 contains only self-clique graphs and the family in Section 4 contains only 2-self-clique graphs. It is a classic result that any clique-Helly graph without dominated vertices is either self-clique or 2-self-clique (Escalante 1973, [7]), and any counterexample to the conjecture cannot contain dominated vertices (since the removal of a dominated vertex from a clique-Helly graph, preserves clique-Hellyness).

A preliminary version of this work appeared in [1] where we showed that one counterexample to the conjecture exists.

\footnotetext{
* Corresponding author at: Dto. de Matemática, FCE-UNLP, La Plata, Argentina.

E-mail addresses: liliana@mate.unlp.edu.ar (L. Alcón), map@xanum.uam.mx (M. Pizaña), gravenna@mate.unlp.edu.ar (G. Ravenna).
} 


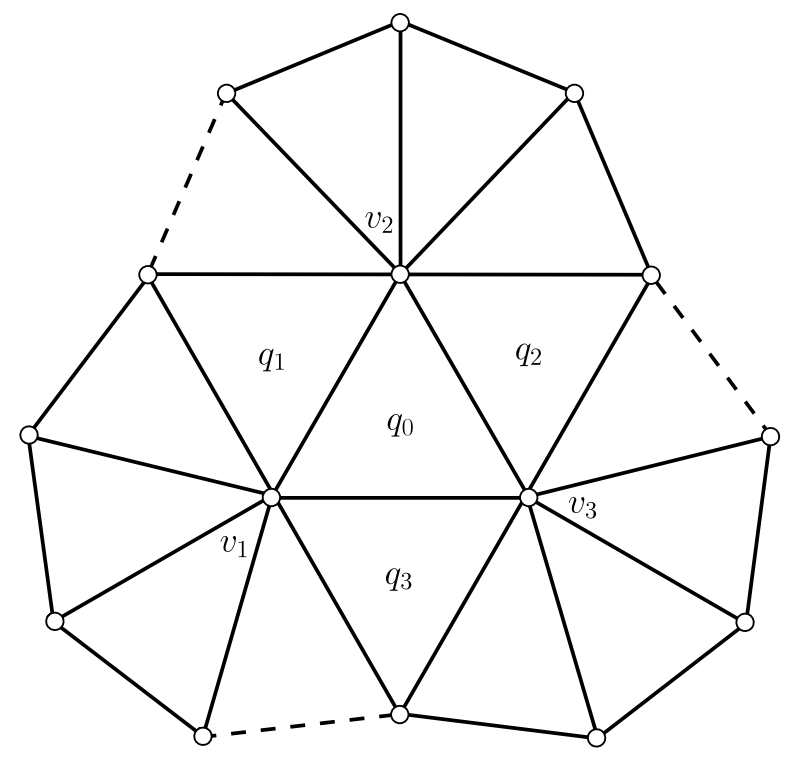

Fig. 1. A partial drawing of a locally $C_{d}$ graph.

\section{Preliminaries}

Our graphs are finite and simple. We identify induced subgraphs with their vertex set, in particular we usually write $x \in G$ instead of $x \in V(G)$. Also, for a vertex $x \in G$, we write $G-x$ instead of $G-\{x\}$. The open and the closed neighborhood of a vertex $x \in G$ are denoted by $N(x)$ and $N[x]$ respectively. The degree of $x$ is the cardinality of $N(x)$. We write $x \simeq y$ when $x$ is adjacent-or-equal to $y$.

The complete graph on $n$ vertices is denoted by $K_{n}$. A clique is a maximal complete subgraph. Let $\mathcal{C}(G)$ be the family of all cliques of $G$. When $\mathcal{C}(G)$ satisfies the Helly property, we say that $G$ is a clique-Helly graph.

Definition 2.1. A graph $G$ is critical clique-Helly if $G$ is clique-Helly and $G-x$ is not clique-Helly for every $x \in G$.

Notice that in terms of the previous definition the conjecture of Dourado, Protti and Szwarcfiter postulates that there are no critical clique-Helly graphs.

The clique graph $K(G)$ of $G$ is the intersection graph of $\mathcal{C}(G)$ : the vertices of $K(G)$ are the cliques of $G$ and two different cliques of $G$ are adjacent in $K(G)$ if and only if they have non-empty intersection. The second clique graph of $G$ is $K^{2}(G)=K(K(G))$. Then the vertices of $K^{2}(G)$ are the cliques of $K(G)$ which are said to be cliques of cliques of $G$. Given a vertex $v$ of a graph $G$, the star of $v$ is the set of all the cliques of $G$ which contain $v$, i.e. $v^{*}=\{q \in V(K(G)): v \in q\}$. Stars of $G$ are not always vertices of $K^{2}(G)$ : They are always complete subgraphs of $K(G)$, but not always maximal. Any clique of cliques of $G$ which is not a star will be said to be a necktie. An example of a necktie is $Q=\left\{q_{0}, q_{1}, q_{2}, q_{3}\right\}$, where $q_{i}$ is the clique formed by the vertices of the corresponding triangles in Fig. 1. We say that $G$ is self-clique if $K(G) \cong G$, and that it is 2-self-clique whenever $K^{2}(G) \cong G 尹 K(G)$.

A cycle in $G$ is a sequence of at least three distinct vertices $v_{1}, v_{2}, \ldots, v_{d}$ of $G$ such that two of them are adjacent in $G$ if and only if they are consecutive in the sequence or they are $v_{1}$ and $v_{d}$. The positive integer $d$ is the length of the cycle. The cycle of length $d$ is denoted by $C_{d}$. A graph $G$ is locally cyclic if each open neighborhood in $G$ induces a cycle, and $G$ is a locally $C_{d}$ graph if $N(v)$ induces a $C_{d}$ for every $v \in G$. The girth $g(G)$ of $G$ is the length of a shortest cycle in $G$ (if $G$ has no cycles, then $g(G)=\infty)$. The local girth of $G$ at a vertex $v \in G, \lg _{v}(G)$, is the girth of the subgraph induced by the open neighborhood of $v$ in $G$, i.e. $\lg _{v}(G)=g(N(v))$. The minimum of these local girths is denoted by $\lg (G)$ and is called the local girth of $G$, i.e.

$$
\lg (G)=\min \left\{\lg _{v}(G): v \in G\right\} .
$$

Theorem 2.2 ([10]). If the local girth of the graph $G$ is greater than 6 (i.e. $\lg (G) \geq 7)$ then $K(G)$ is clique-Helly.

For $d \geq 7$ and $G$ a locally $C_{d}$ graph, a detailed analysis of the cliques and cliques of cliques of $G$ was done in [10]. We transcribe here the most relevant properties for our purposes (which can all be verified straightforwardly): 
Remark 2.3 ([10], Section 3.1). If $d \geq 7$, and $G$ is a locally $C_{d}$ graph, then:

1. All the cliques of $G$ are triangles.

2. For every vertex $v \in G, v^{*}$ is a clique of cliques of $G$.

3. For every triangle $T=\left\{v_{1}, v_{2}, v_{3}\right\}$ of $G$, there is a necktie $Q_{T}=\{q \in K(G):|q \cap T| \geq 2\}$, which is a clique of cliques of $G$ and it is always of the form $Q_{T}=\left\{q_{0}, q_{1}, q_{2}, q_{3}\right\}$ (see Fig. 1).

4. Every clique of cliques of $G$ is either a star $v^{*}$ or a necktie $Q_{T}$.

5. In $K^{2}(G), v_{1}^{*} \simeq v_{2}^{*}$ if and only if $v_{1} \simeq v_{2}$ in $G$.

6. In $K^{2}(G), Q_{T} \simeq Q_{T^{\prime}}$ if and only if either $T$ and $T^{\prime}$ share an edge, or they share a vertex and there is an edge joining a vertex of $T \backslash T^{\prime}$ with a vertex of $T^{\prime} \backslash T$.

7. In $K^{2}(G), v^{*} \simeq Q_{T}$ if and only if $v \in \cup Q_{T}=\cup_{i=0}^{3} q_{i}$ where $Q_{T}=\left\{q_{0}, q_{1}, q_{2}, q_{3}\right\}$.

Brown and Connelly proved in [3] that there exists at least one finite locally $C_{d}$ graph for each $d \geq 3$. Larrión, Neumann-Lara and Pizaña obtained the next theorem extending the result of Brown and Connelly for $d \geq 7$.

Theorem 2.4 ([10]). Let $d$ be any integer greater than or equal to 7. Then there are infinitely many non-isomorphic locally $C_{d}$ graphs.

In the strong product of graphs, $G \otimes H$, two vertices $\left(g_{1}, h_{1}\right),\left(g_{2}, h_{2}\right) \in V(G \otimes H)=V(G) \times V(H)$ are adjacent-or-equal whenever $g_{1} \simeq g_{2}$ in $G$ and $h_{1} \simeq h_{2}$ in $H$. We refer the reader to [9] for the known results on the strong product. A classic result on clique graphs (Neumann-Lara, 1978) states that the clique operator distributes over the strong product of graphs:

Theorem $2.5([11]) . K(G \otimes H) \cong K(G) \otimes K(H)$.

\section{The self-clique family}

Let $r, s, t \geq 4$. Take $G(r, s, t)=C_{r} \otimes C_{s} \otimes C_{t}$. We claim that these graphs are the sought self-clique counterexamples:

Theorem 3.1. All the graphs $G(r, s, t)$ are self-clique critical clique-Helly graphs.

Proof. Certainly they are all self-clique since, by Theorem 2.5, we have $K(G(r, s, t))=K\left(C_{r} \otimes C_{s} \otimes C_{t}\right) \cong K\left(C_{r}\right) \otimes K\left(C_{s}\right) \otimes$ $K\left(C_{t}\right) \cong C_{r} \otimes C_{s} \otimes C_{t}=G(r, s, t)$.

Evidently, every $C_{d}$ is clique-Helly. Let us see that the strong product of clique-Helly graphs is again clique-Helly:

Suppose $X$ and $Y$ are clique-Helly graphs. The cliques of $X \otimes Y$ are of the form $q=q_{1} \times q_{2}$ with $q_{1} \in \mathcal{C}(X)$ and $q_{2} \in \mathcal{C}(Y)$. Now assume you have a pairwise intersecting family of cliques of $X \otimes Y$, namely: $q^{1}=q_{1}^{1} \times q_{2}^{1}, q^{2}=q_{1}^{2} \times q_{2}^{2}, \ldots, q^{m}=$ $q_{1}^{m} \times q_{2}^{m}$. It follows that $q_{1}^{1}, q_{1}^{2}, \ldots, q_{1}^{m}$ are pairwise intersecting cliques in $X$ and $q_{2}^{1}, q_{2}^{2}, \ldots, q_{2}^{m}$ are pairwise intersecting cliques in $Y$. Since $X$ and $Y$ are clique-Helly, there are some vertices $x \in \cap_{i=1}^{m} q_{1}^{i} \subseteq X$ and $y \in \cap_{i=1}^{m} q_{2}^{i} \subseteq Y$. Clearly, $(x, y)$ belongs to the total intersection of $q^{1}, q^{2}, \ldots, q^{m}$ and therefore $X \otimes Y$ is clique-Helly.

It follows that $G(r, s, t)=C_{r} \otimes C_{s} \otimes C_{t}$ is clique-Helly.

Now we will show that $G(r, s, t)-x$ is not clique-Helly for every vertex $x$. Since $G(r, s, t)$ is clearly vertex-transitive, it is sufficient for us to prove it for any particular vertex $x$. Assume the vertices of each cycle $C_{d}$ are numbered as $\{1,2, \ldots, d\}$ for $d \in\{r, s, t\}$ and take $x=(2,2,2) \in G(r, s, t)$. Define $H=G(r, s, t)-x$. Now in $G(r, s, t)$ take the cliques $q_{1}=\{2,3\} \times\{1,2\} \times\{1,2\}, q_{2}=\{1,2\} \times\{2,3\} \times\{1,2\}, q_{3}=\{1,2\} \times\{1,2\} \times\{2,3\}$. The corresponding cliques in $H, \bar{q}_{i}=q_{i} \cap H=q_{i}-(2,2,2)$ for $i=1,2,3$, are pairwise intersecting (each $q_{i} \cap q_{j}$ contains one of the following vertices: $(1,2,2),(2,1,2)$ or $(2,2,1))$ but have no common total intersection: the only vertex in $q_{1} \cap q_{2} \cap q_{3}$ in $G(r, s, t)$ is $(2,2,2)$ which is not present in $H$. It follows that $H$ is not clique-Helly.

\section{The 2-self-clique family}

Theorem 4.1. Let $G$ be a locally $C_{d}$ graph with $d \geq 7$. Then $K(G)$ is a critical clique-Helly graph.

Proof. Since $G$ is locally $C_{d}$ graph, the local girth of $G$ equals $d \geq 7$, therefore, by Theorem $2.2, K(G)$ is clique-Helly.

Let $q_{0}$ be any vertex of $K(G)$ (a clique of $G$ ). We will prove that $K(G)-q_{0}$ is not clique-Helly. By Remark 2.3(1) every clique in $G$ is a triangle. Without loss of generality assume $q_{0}=\left\{v_{1}, v_{2}, v_{3}\right\}$ as in Fig. 1 .

Consider the following cliques of $K(G)-q_{0}: v_{1}^{*}-q_{0}, v_{2}^{*}-q_{0}$ and $v_{3}^{*}-q_{0}$. We claim these three cliques are pairwise intersecting but the intersection of all three of them is empty: indeed, the vertices of $K(G)-q_{0}$ corresponding to the cliques $q_{1}, q_{2}$ and $q_{3}$ of $G$ (as in Fig. 1) belong to $v_{1}^{*} \cap v_{2}^{*}, v_{2}^{*} \cap v_{3}^{*}$ and $v_{3}^{*} \cap v_{0}^{*}$, respectively. Finally, assume in order to obtain a contradiction that a vertex $q$ of $K(G)-q_{0}$ belongs to $v_{1}^{*} \cap v_{2}^{*} \cap v_{3}^{*}$, then, by definition of these sets, $q$ is a clique of $G$ such that $v_{i} \in q$ for $i \in\{1,2,3\}$. Thus, $q=\left\{v_{1}, v_{2}, v_{3}\right\}=q_{0}$ which contradicts our assumption that $q$ is a vertex of $K(G)-q_{0}$. Hence $K(G)-q_{0}$ is not clique-Helly. 
Theorem 4.2. Let $G_{1}$ and $G_{2}$ be two non-isomorphic locally $C_{d}$ graphs. Then $K\left(G_{1}\right)$ and $K\left(G_{2}\right)$ are also non-isomorphic.

Proof. Assume, by way of contradiction, that $K\left(G_{1}\right) \cong K\left(G_{2}\right)$. Then we also have $K^{2}\left(G_{1}\right) \cong K^{2}\left(G_{2}\right)$. Now, by Remark $2.3(4)$, the vertices of $K^{2}\left(G_{1}\right)$ (and those of $K^{2}\left(G_{2}\right)$ ) are either stars or neckties. By Remark 2.3(5-7), stars have degree 3d ( $d$ of the neighbors are stars and $2 d$ are neckties) and neckties have degree 15 (6 stars and 9 neckties), see Fig. 1 . Hence, any isomorphism $\phi: K^{2}\left(G_{1}\right) \rightarrow K^{2}\left(G_{2}\right)$ must map stars onto stars bijectively. Let $S_{1}$ and $S_{2}$ be the subgraphs of $K^{2}\left(G_{1}\right)$ and $K^{2}\left(G_{2}\right)$ induced by the stars of $G_{1}$ and $G_{2}$ (respectively). Hence the restriction of $\phi, \phi^{\prime}: S_{1} \rightarrow S_{2}$, is still an isomorphism. But, by Remark 2.3(5), $G_{1} \cong S_{1}$ and $G_{2} \cong S_{2}$. It follows that $G_{1} \cong S_{1} \cong S_{2} \cong G_{2}$, contrary to our hypothesis.

Theorem 4.3. There are infinitely many 2-self-clique critical clique-Helly graphs.

Proof. Let $G$ be a locally $C_{d}$ graph and $H=K(G)$. It follows by Theorem 4.1 , that $H$ is a critical clique-Helly graph. By Theorem 2.4, there are infinitely many such examples, all of them non-isomorphic to each other by Theorem 4.2 .

The fact that these examples are not self-clique follows from vertex degree comparisons: As in the proof of Theorem 4.2, the vertices of $K(H)=K^{2}(G)$ have a degree which is either $3 d$ or 15 , but the degree of all vertices of $H=K(G)$ is $3 d-6$ (see Fig. 1 and Remark 2.3(1)). It follows that $K(H) \approx H$ and hence $H$ is not self-clique. By the result of Escalante mentioned in the introduction, $H$ is 2 -self-clique.

\section{Acknowledgments}

We are grateful to the anonymous reviewers whose comments helped us improve the presentation of this paper.

This work is partially supported by grant A1-S-45528, SEP-CONACyT, Mexico; and by PIP 11220150100703, CONICET, Argentine.

\section{References}

[1] L. Alcón, M. Pizaña, G. Ravenna, On the existence of critical clique-Helly graphs, in: LAGOS'17 - IX Latin and American Algorithms, Graphs and Optimization Symposium, Electron. Notes Discrete Math. 62 (2017) 231-236, http://dx.doi.org/10.1016/j.endm.2017.10.040.

[2] C. Berge, The Helly property, Southeast Asian Bull. Math. 1 (1977) 16-19.

[3] M. Brown, R. Connelly, On graphs with a constant link, in: New Directions in the Theory of Graphs (Proc. Third Ann Arbor Conf., Univ. Michigan, Ann Arbor, Mich., 1971), Academic Press, New York, 1973, pp. 19-51.

[4] L. Danzer, B. Grünbaum, V. Klee, Helly's theorem and its relatives, in: Proc. Sympos. Pure Math. Vol. VII, Amer. Math. Soc., Providence, RI, 1963, pp. 101-180.

[5] M.C. Dourado, F. Protti, J.L. Szwarcfiter, Computational aspects of the Helly property: a survey, J. Braz. Comput. Soc. 12 (2006) 7-33, http://dx.doi.org/10.1007/BF03192385.

[6] J. Eckhoff, Helly, Radon, and Carathéodory type theorems, in: Handbook of Convex Geometry, Vol. A, B, North-Holland, Amsterdam, 1993, pp. 389-448.

[7] F. Escalante, Über iterierte Clique-Graphen, Abh. Math. Semin. Univ. Hambg. 39 (1973) 59-68.

[8] J.E. Goodman, R. Pollack, R. Wenger, Geometric transversal theory, in: New Trends in Discrete and Computational Geometry, in: Algorithms Combin., vol. 10, Springer, Berlin, 1993, pp. 163-198, http://dx.doi.org/10.1007/978-3-642-58043-7_8.

[9] R. Hammack, W. Imrich, S. Klavžar, Handbook of Product Graphs, second edition, in: Discrete Mathematics and its Applications (Boca Raton), CRC Press, Boca Raton, FL, 2011.

[10] F. Larrión, V. Neumann-Lara, M.A. Pizaña, Whitney triangulations, local girth and iterated clique graphs, Discrete Math. 258 (2002) 123-135, http://dx.doi.org/10.1016/S0012-365X(02)00266-2.

[11] V. Neumann-Lara, CNRS, On clique-divergent graphs, in: Problèmes combinatoires et théorie des graphes (Colloq. Internat. CNRS, Univ. Orsay, Orsay, 1976), in: Colloq. Internat. CNRS, vol. 260, Paris, 1978, pp. 313-315. 